\title{
THE LOW-MASS MEMBERS OF THE URSA MAJOR ASSOCIATION
}

\author{
Jonathan Gagné, ${ }^{1}$ Jacqueline K. Faherty, ${ }^{2}$ and Mark Popinchalk ${ }^{2,3}$ \\ ${ }^{1}$ Planétarium Rio Tinto Alcan, Espace pour la Vie, 4801 av. Pierre-de Coubertin, Montréal, Québec, Canada \\ ${ }^{2}$ Department of Astrophysics, American Museum of Natural History, Central Park West at 79th St., New York, NY 10024, USA \\ ${ }^{3}$ The Graduate Center, City University of New York, New York, NY 10016, USA
}

Keywords: methods: data analysis — stars: kinematics and dynamics — proper motions 
The Ursa Major association (Eggen 1992) is a nearby ( $25 \mathrm{pc}$ ) collection of ten F2 to A5-type stars (King et al. 2003; Gagné et al. 2018c), aged $414 \pm 23 \mathrm{Myr}$ (Jones et al. 2015), distributed in a small core mostly consisting of stars in the Big Dipper asterism, with a scatter in Galactic positions $(X Y Z)$ of about 1-3 pc. It is accompanied by a stream of 42 known stars (King et al. 2003) with a wider range in spectral types and a much wider $X Y Z$ scatter of $25-40 \mathrm{pc}$. The core is slightly better localized in Galactic velocities $U V W$ with a scatter of $1-3 \mathrm{~km} \mathrm{~s}^{-1}$, compared with $2.5-4 \mathrm{~km} \mathrm{~s}^{-1}$ for the stream members. Despite it being the nearest open cluster to the Sun, its population of low-mass members is still largely missing (only $3 \mathrm{~K}$-type stars and 1 M-type star are part of the stream), likely because they have been scattered away over time. Even most recent surveys based on URAT-South (Finch et al. 2018), RECONS (Winters et al. 2015) and Gaia DR2 (Gaia Collaboration et al. 2018) only identified two additional M-type candidate members (2MASS J13445785+5528219 and 2MASS J12153937+5239088; Gagné et al. 2018a). Preliminary surveys using only the kinematic portion of the BANYAN $\Sigma$ algorithm (Gagné et al. 2018c) to identify lower-mass candidates with a larger $X Y Z$ spread, but centered on the same Gaussian model of known members in $U V W$, did not allow us to uncover a significant number of low-mass members, leading us to hypothesize they might have been scattered beyond recovery.

A recent study by Kounkel \& Covey (2019) used a hierarchical clustering algorithm applied to Gaia DR2 data to uncover over-densities in kinematic observable space (sky position, proper motion, and parallax) within $1 \mathrm{kpc}$ and $30^{\circ}$ of the Galactic plane. They uncovered 1640 localized or extended over-densities, the latter of which they called strings, and estimated isochronal ages for all of them. Because their method is based in direct observables rather than $X Y Z U V W$-the latter three cannot be calculated for the majority of Gaia DR2 entries which lack a radial velocity measurement- they did not identify over-densities in the immediate Solar neighborhood, within about $70 \mathrm{pc}$ around the Sun, except for the very dense Hyades. For this reason, they have not recovered any of the classical members of Ursa Major, neither the core or stream, as they all lie within this neighborhood.

While inspecting the nearest $(<200 \mathrm{pc})$ and youngest $(<1 \mathrm{Gyr}) 73$ groups identified by Kounkel \& Covey (2019), we noticed that five of them (Theia 906, 908, 1008, 1009 and 1091), totaling 1599 stars, clustered around a similar $U V W$ distribution to the known Ursa Major core and stream, while extending spatially along two large tails that reach out to almost $350 \mathrm{pc}$ from the Sun (see Figure 1). All but Theia 1091 are designated as strings. Despite their much wider distribution in space velocities $\left(18,33\right.$, and $11 \mathrm{~km} \mathrm{~s}^{-1}$ in $U, V$ and $W$ respectively), they have been assigned isochronal ages in the range 503-849 Myr by Kounkel \& Covey (2019), all of which could be consistent with real members of Ursa Major given that these isochronal ages are based on models with significant systematics (e.g., see the discussion of Gagné et al. 2018b), and have not been corrected for interstellar extinction. The wider spread in space velocities, especially along the direction of Galactic rotation $V$, could indicate that these arms are tidal tails well into the process of dissipation, but not exactly beyond recovery as we had previously hypothesized. It is interesting to note that the proposed Sirius supercluster also identified by Eggen (1992) displays a similar distribution in $U V W$ space to the Theia groups from Kounkel \& Covey (2019) discussed here, and could be related although it may be a contaminated sample-Eggen (1992) noted that it seems to consist of some stars aged $\sim 630 \mathrm{Myr}$ and others $\sim 1$ Gyr.

The Gaia DR2 absolute $G$ magnitude versus $G-G_{\mathrm{RP}}$ color diagram of the Theia groups discussed here seems consistent with a coeval, 500 Myr-old stellar population: it ends abruptly at the upper main sequence around $G-G_{\mathrm{RP}}=0$, and its most massive component, HD 199713, is a B9 star (Wright et al. 2003), corresponding to an estimated mass of $\sim 2.8 M_{\odot}$ from the spectral type-mass relations of Pecaut \& Mamajek (2013). Other mass estimates for HD 199713 based on a comparison of its Gaia DR2 photometry to models or empirical relations from eclipsing binaries range from $2.9_{-0.4}^{+0.3} M_{\odot}$ (Anders et al. 2019) to $3.0 \pm 0.4 M_{\odot}$ (Stassun et al. 2019). These mass estimates correspond to stars that spend 460-580 Myr on the main sequence according to the MIST models (Choi et al. 2016), consistent with current age estimates for Ursa Major. The lowest-mass portion of the color-magnitude diagram contains 990 stars with $G-G_{\mathrm{RP}}$ between 1.0 and $1.4 \mathrm{mag}$, corresponding to spectral types M0-M5, and are not significantly over-luminous compared to the distribution of older field stars, also consistent with the Ursa Major age. Both the Ursa Major stream defined by King et al. (2003) and the Sirius supercluster described by Eggen (1992) have similar color-magnitude diagram sequences with consistent main-sequence turn-off points. Only one Sirius supercluster star is bluer, $\beta$ Aur (spectral type A1, see Gray et al. 2003), but this is likely due to it being an eclipsing binary. The mass of its individual components have been measured by Belikov (1995) at $2.35 \pm 0.03 M_{\odot}$ and $2.27 \pm 0.03 M_{\odot}$, and are therefore less massive than HD 199713 and consistent with the quoted age of Ursa Major. 
The aforementioned Kounkel \& Covey (2019) groups contain 20 white dwarfs based on their position in the Gaia DR2 color-magnitude diagram: 16 of them are clearly too old for bearing any relation to Ursa Major based on a comparison with the Montréal C/O core cooling tracks ${ }^{1}$. Further, all of these contaminant white dwarfs are located within 200 pc, suggesting that the more distant ones have simply not been uncovered because of their faintness. Combining this number with local number space densities for white dwarfs $\left(4.49 \pm 0.38 \times 10^{-3}\right.$ objects pc $^{-3}$; Hollands et al. 2018) and main-sequence stars $\left(98.4 \pm 6.8 \times 10^{-3}\right.$ objects pc $^{-3}$; Kirkpatrick et al. 2012) suggests we might expect a total of $\sim 520$ main-sequence false-positive members, or a contamination rate of about $\sim 33 \%$. We note that most of the white dwarf interlopers are located in Theia 906 (6 interlopers) and Theia 908 (also 6 interlopers), indicating they may be more contaminated - consistent with their wider $U V W$ distributions. The remaining are in Theia 1008 (3 interlopers) and Theia 1091 (1 interloper). Four additional white dwarfs (WDJ235833.30+510815.54, WDJ233848.60+404803.82, WDJ232227.23+394456.36, and WDJ121521.73-523646.04) have temperatures in the range $8000 \mathrm{~K}-24000 \mathrm{~K}$, masses in the range 1.00-1.27 $M_{\odot}$ (Gentile Fusillo et al. 2019), and total (main-sequence plus cooling) ages in the range 400-800 Myr, based on non-dereddened Gaia DR2 color-magnitude positions.

Most of the members in these candidate tidal tails are distributed between -20 to $+20^{\circ}$ in Galactic longitude, indicating that there might not be many candidates left to be recovered beyond the -30 to $+30^{\circ}$ range that was surveyed by Kounkel \& Covey (2019). Stars at the upper main sequence of the color-magnitude diagram have a range of distances from $73 \mathrm{pc}$ to $330 \mathrm{pc}$, compared to $67-330 \mathrm{pc}$ for the full sample: this indicates no obvious distancedependent contamination in their sample of lower-mass stars. It can however be expected that more members may be left to uncover within 70 pc of the Sun, where the Kounkel \& Covey (2019) algorithm was less sensitive. We have observed no clear correlation between the spatial $X Y Z$ and kinematic $U V W$ distribution of members, meaning that any search for nearby members will likely require using a very wide net in $U V W$ space, and may suffer a high rate of contamination despite the unusual kinematics of Ursa Major compared with the Solar neighborhood. This also explains why our previous searches based on the $U V W$ distribution of the core members failed to uncover anything.

More work is clearly needed to disentangle the relations between the different groups discussed here, and to assess whether the large structures recovered by Kounkel \& Covey (2019) are really related to Ursa Major, and where the Eggen (1992) Sirius supercluster, and the King et al. (2003) Ursa Major stream, fit into that picture. It is likely, for example, that some members at the extremes of the $U V W$ distributions of Theia 906 and 908 suffer from low-quality measurements or correspond to contaminants. Using metrics of stellar activity and rotation periods from the TESS mission (Ricker et al. 2015) will be useful to corroborate membership although only for the nearest members (e.g., see Curtis et al. 2019), and a more detailed study of individual members will be useful to further investigate the relation between the Kounkel \& Covey (2019) groups discussed here and Ursa Major. Gaia DR3 will be useful in this investigation as it will likely contain radial velocity measurements for several stars discussed here. It will also be interesting to determine why Ursa Major might have much larger tidal tails with a wider space velocity spread than the similarly-aged Coma Ber (Silaj \& Landstreet 2014) which tidal tails were recently discovered by Tang et al. (2019). Studying the four white dwarfs discussed above in more details will also be useful to better age-date this population.

We would like to thank Eric E. Mamajek and Andrew W. Mann for useful comments and discussion. This research made use of data from the ESA mission Gaia, processed by the Gaia Data Processing and Analysis Consortium whose funding has been provided by national institutions, in particular the institutions participating in the Gaia Multilateral Agreement.

Software: BANYAN $\Sigma$ (Gagné et al. 2018c).

\footnotetext{
${ }^{1}$ Computed similarly as the pure C core models described in Fontaine et al. (2001), and available at http://www.astro.umontreal.ca/ $\sim$ bergeron/CoolingModels/
} 


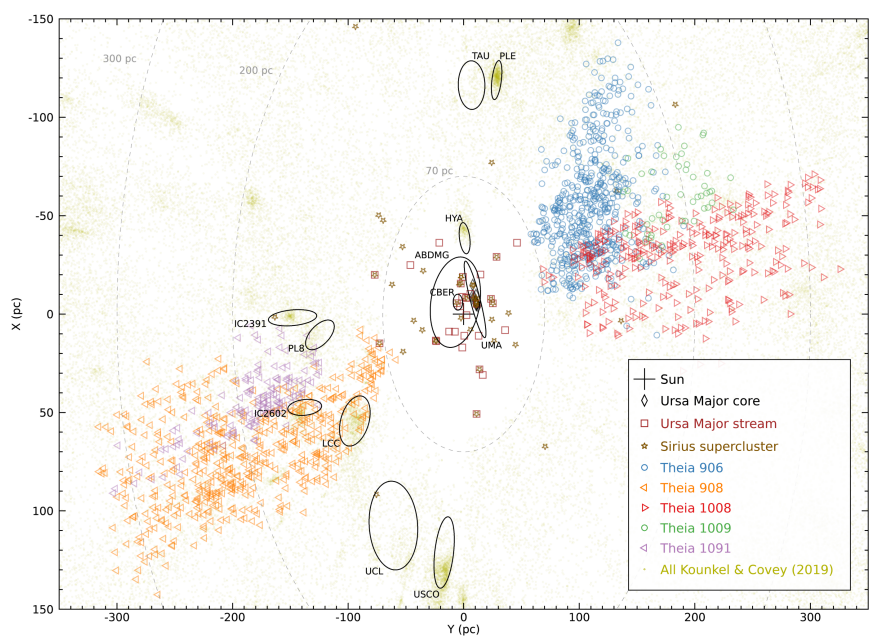

(a) $X Y$

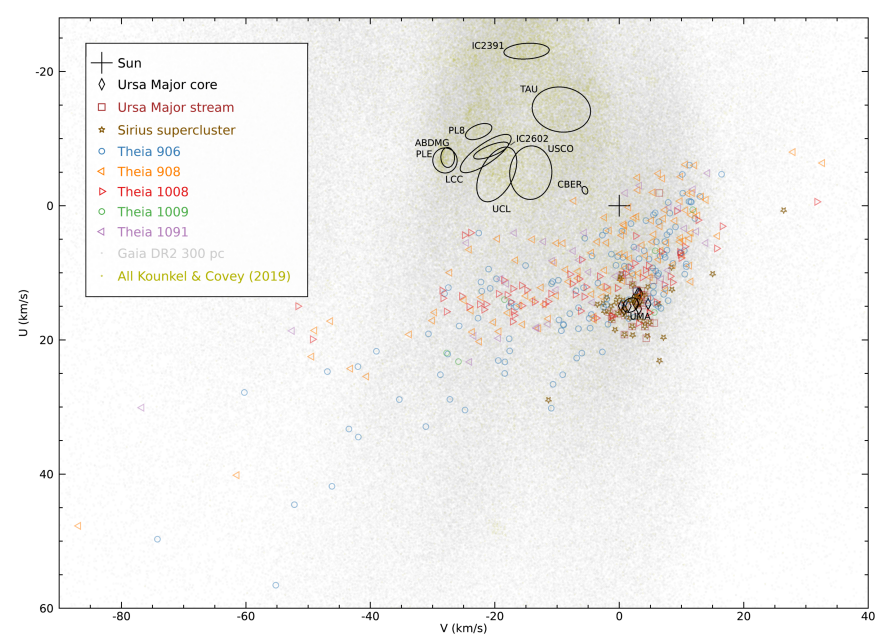

(b) $U V$

Figure 1. Galactic positions $X Y$ and space velocities $U V$ of the Kounkel \& Covey (2019) groups that may constitute tidal tails to the Ursa Major group. They are located between $\sim 70 \mathrm{pc}$ and $\sim 330 \mathrm{pc}$, and have space velocities similar to Ursa Major although more scattered, especially in the direction of Galactic rotation $(V)$. Ellipses represent the multivariate Gaussian models of BANYAN $\Sigma$, and indicate the loci of members for IC 2391, Platais 8, IC 2602, Lower Centaurus Crux (LCC), Upper Centaurus Lupus (UCL), Upper Scorpius (USCO), the Taurus-Auriga star-forming region (TAU), the Pleiades association (PLE), the Hyades association (HYA), the AB Doradus moving group (ABDMG), the Ursa Major association (UMA), and the Coma Berenices association (CBER). For more details on these models and associations, see Gagné et al. (2018c). Data behind figures is available as online-only material.

\section{REFERENCES}

Anders, F., Khalatyan, A., Chiappini, C., et al. 2019, Astronomy and Astrophysics, 628, A94

Belikov, A. N. 1995, Bulletin d'Information du Centre de Donnees Stellaires, 47, 9

Choi, J., Dotter, A., Conroy, C., et al. 2016, The Astrophysical Journal, 823, 102

Curtis, J. L., Agüeros, M. A., Mamajek, E. E., Wright, J. T., \& Cummings, J. D. 2019, The Astronomical Journal, 158, 77
Eggen, O. J. 1992, Astronomical Journal, 104, 1493

Finch, C. T., Zacharias, N., \& Jao, W.-C. 2018, The Astronomical Journal, 155, 176

Fontaine, G., Brassard, P., \& Bergeron, P. 2001, The Publications of the Astronomical Society of the Pacific, 113,409

Gagné, J., Faherty, J. K., \& Fontaine, G. 2018a, Research Notes of the American Astronomical Society, 2, 9 
Gagné, J., Fontaine, G., Simon, A., \& Faherty, J. K. 2018b, The Astrophysical Journal Letters, 861, L13

Gagné, J., Mamajek, E. E., Malo, L., et al. 2018c, The Astrophysical Journal, 856, 23

Gaia Collaboration, Brown, A. G. A., Vallenari, A., et al. 2018, Astronomy \& Astrophysics, 616, A1

Gentile Fusillo, N. P., Tremblay, P.-E., Gänsicke, B. T., et al. 2019, Monthly Notices of the Royal Astronomical Society, 482, 4570

Gray, R. O., Corbally, C. J., Garrison, R. F., McFadden, M. T., \& Robinson, P. E. 2003, The Astronomical Journal, 126, 2048

Hollands, M. A., Tremblay, P. E., Gänsicke, B. T., Gentile-Fusillo, N. P., \& Toonen, S. 2018, Monthly Notices of the Royal Astronomical Society, 480, 3942

Jones, J., White, R. J., Boyajian, T., et al. 2015, The Astrophysical Journal, 813, 58

King, J. R., Villarreal, A. R., Soderblom, D. R., Gulliver, A. F., \& Adelman, S. J. 2003, The Astronomical Journal, 125,1980
Kirkpatrick, D. J., Gelino, C. R., Cushing, M. C., et al. 2012, The Astrophysical Journal, 753, 156

Kounkel, M., \& Covey, K. 2019, arXiv.org, 122

Pecaut, M. J., \& Mamajek, E. E. 2013, The Astrophysical Journal Supplement, 208, 9

Ricker, G. R., Winn, J. N., Vanderspek, R., et al. 2015, Journal of Astronomical Telescopes, 1, 014003

Silaj, J., \& Landstreet, J. D. 2014, Astronomy and Astrophysics, 566, A132

Stassun, K. G., Oelkers, R. J., Paegert, M., et al. 2019, The Astronomical Journal, 158, 138

Tang, S.-Y., Pang, X., Yuan, Z., et al. 2019, The Astrophysical Journal, 877, 12

Winters, J. G., Henry, T. J., Lurie, J. C., et al. 2015, The Astronomical Journal, 149, 5

Wright, C. O., Egan, M. P., Kraemer, K. E., \& Price, S. D. 2003, The Astronomical Journal, 125, 359 